\title{
On the Euler-Bernoulli and Timoshenko-Ehrenfest beam theories under fractional calculus approach
}

\author{
José Ivelton S. Lustosal \\ IMECC/Unicamp, Campinas, SP \\ Flávio de Campos Bannwart作 \\ FEM/Unicamp, Campinas, SP \\ Edmundo Capelas de Oliveira $\$$ \\ IMECC/Unicamp, Campinas, SP
}

\begin{abstract}
General fractional solutions for the Euler-Bernoulli and Timoshenko-Ehrenfest differential equations are deduced for the case of a simply supported beam submitted to uniformly distributed static load. Both solutions are confronted against Ansys software results, taken as reference, leading to the corresponding particular fractional solutions. As expected, both particular solutions converge to the Ansys results for small deflections and beam with high-aspect ratio, while, for opposite geometrical features, the fractional Timoshenko-Ehrenfest solution performs slightly better, kept the linear elasticity. The shear effects are realized in the fractional order transformation of the Euler-Bernoulli model; besides, from comparative analysis, other physical effects in the structure may be present in the fractional order.

Resumo. Soluções fracionárias gerais para as equações diferenciais de Euler-Bernoulli e TimoshenkoEhrenfest são deduzidas para o caso de uma viga biapoiada submetida a uma carga estática uniformemente distribuída. Ambas as soluções são confrontadas com os resultados do software Ansys, tomado como referência, levando às soluções fracionárias particulares correspondentes. Como esperado, ambas as soluções específicas convergem para os resultados do Ansys para pequenas deflexões e vigas de alta razão de aspecto, enquanto que, na geometria oposta, a solução fracionária de Timoshenko-Ehrenfest tem um desempenho ligeiramente melhor, mantida a elasticidade linear. Os efeitos de cisalhamento são constatados na transformação de ordem fracionária do modelo de EulerBernoulli; além disso, a partir da análise comparativa, outros efeitos físicos na estrutura podem estar presentes na ordem fracionária.
\end{abstract}

Keywords. Euler-Bernoulli, Timoshenko,Timoshenko-Ehrenfest, Ansys, Fractional calculus.

\section{Introduction}

In structural engineering, two beam theories for linear elastic regime are of fundamental importance: Euler-Bernoulli (EB) and Timoshenko-Ehrenfest (TE). EB beam theory works under the premise that for small deflections of a high-aspect ratio (length to principal transversal dimension) prismatic beam submitted to a lateral load the cross sections remain flat and perpendicular to the neutral plane. TE beam theory, on the other hand, likewise imposes the cross sections flatness for

\footnotetext{
${ }^{1}$ j228022@dac.unicamp.br.

2 fcbannwart@fem.unicamp.br.

3 capelas@unicamp.br.
} 
small deflections, however not necessarily perpendicular to the neutral plane; this comes down to stating that such a theory includes the shear effects, increasingly present with lower aspect ratio, which leads to more accurate modeling for thick beams. EB is therefore a particular case of TE, both analytical integer order models restricted to simple and linear systems.

In this work we propose and solve fractional models for both beam theories using corresponding Ansys numerical solutions as reference for a specific example. The resulting fractional models, as expected, circumvent most of the linear limitations of the original models, ending up closer to the Ansys results. Moreover, as expected as well, the fractional TE models (FTE) perform slightly better than the fractional EB ones (FEB) due to its shear effects consideration, already intrinsic to the TE beam theory; the EB beam theory does not predict such effects. Departing from those fractional models, the shear effects, necessarily present in the Ansys model, remain represented in the transformation from TE to FTE, and start to be represented in the transformation from BE to FEB, as a clear improvement. Therefore, the fractional order is a measure of improvement as much as it deviates from the original integer order, so that to approach the behavior of real systems. For this reason, the improvement in FTE is lower than in FEB. Comparing and analyzing those results lead to the verification of the shear effects repercussion in the fractional order and enable the search for other physical effects possibly contemplated in this way. So far, we did not find any works directed to such a kind of investigation.

\section{Analytical development}

A simply supported beam is chosen for the investigation. The beam is prismatic, homogeneous, isotropic, linearly elastic, and $L$ in length. The bending moment and shear force are represented by $M$ and $V$, respectively. $\vec{q}$ is the uniformly distributed load along its length.

According to 5], BE beam theory states that

$$
E I \frac{d^{4} v(x)}{d x^{4}}=-q(x)
$$

where $E$ is the Young's modulus and $I$ is the rectangular moment of inertia of the cross section.

For our specific problem, applying the boundary conditions $v(0)=v(L)=0$ and $v^{\prime}\left(\frac{L}{2}\right)=$ $M(0)=0$, the solution for the bending strain results

$$
v(x)=\frac{q}{E I}\left(\frac{-x^{4}}{24}+\frac{L x^{3}}{12}-\frac{L^{3} x}{24}\right) .
$$

\subsection{Fractional Euler-Bernoulli solution}

The following fractional differential equation is similar to the Eq.(1), which models the specific problem studied here. We employ the Caputo fractional derivative, defined in [2], [4] and [6], for example. The fractional model proposed for such a solution is the fractional ordinary differential equation of order $\alpha$

$$
\frac{d^{\alpha} v}{d x^{\alpha}}=-\frac{q}{E I}
$$

with $4 \leq \alpha<5$ and $v=v(x)$.

For $m \leq \alpha<m+1$ with $\alpha$ non-integer and $m$ integer, the Laplace transform for derivatives of order $\alpha$ is given by 6

$$
L\left[f^{(\alpha)}(x)\right]=s^{\alpha} F(s)-\sum_{k=1}^{m}\left[s^{\alpha-k} f^{(k-1)}(0)\right],
$$


where $F(s)$ is the Laplace transform of $f(x)$.

Applying Eq.(4) in Eq.(3), we have

$$
s^{\alpha} V(s)-s^{\alpha-1} v(0)-s^{\alpha-2} v^{\prime}(0)-s^{\alpha-3} v^{\prime \prime}(0)-s^{\alpha-4} v^{\prime \prime \prime}(0)=-\frac{q}{E I} \frac{1}{s} .
$$

From the boundary conditions, we have $v(0)=0$. Thus, taking $v^{\prime}(0)=k_{1}, v^{\prime \prime}(0)=k_{2}$ and $v^{\prime \prime \prime}(0)=k_{3}$, replacing in Eq.55 and rearranging the terms, we get

$$
V(s)=-\frac{q}{E I} \frac{1}{s^{\alpha+1}}+\frac{1}{s^{2}} k_{1}+\frac{1}{s^{3}} k_{2}+\frac{1}{s^{4}} k_{3} .
$$

To obtain the solution in the $x$ variable, the application of the inverse Laplace transform in Eq. (6) results in

$$
v(x)=-\frac{q}{E I \Gamma(\alpha+1)} x^{\alpha}+k_{1} x+\frac{k_{2}}{2} x^{2}+\frac{k_{3}}{6} x^{3} .
$$

Since for the case study $v(L)=0, v^{\prime}\left(\frac{L}{2}\right)=0$ and $M(0)=0$, we get

$$
v(x)=\frac{q}{E I}\left[\frac{-x^{\alpha}}{\Gamma(\alpha+1)}+\frac{4 L^{\alpha-3}}{2^{\alpha-1} \Gamma(\alpha+1)}\left(2^{\alpha-1}-\alpha\right) x^{3}+\frac{L^{\alpha-1}}{2^{\alpha-1} \Gamma(\alpha+1)}\left(4 \alpha-3.2^{\alpha-1}\right) x\right] .
$$

Employing $\alpha=4$ into Eq. (8) and taking into account that for $n \in \mathbb{N}$ we have $\Gamma(n+1)=n$ !, the integer solution is recovered in Eq. (2p).

From the development used to obtain Eq. (8) and applying the boundary conditions $M(0)=$ $M(L)=0$, the fractional solution of the bending moment results

$$
M(x)=\frac{q(\alpha-1)}{\Gamma(\alpha)}\left(-x^{\alpha-2}+L^{\alpha-3} x\right) .
$$

For $\alpha=4$,

$$
M(x)=\frac{-q x^{2}}{2}+\frac{q L}{2} x .
$$

Analogously, we obtain the expression for the modified (fractional) transversal shear force. Employing the boundary condition $V\left(\frac{L}{2}\right)=0$ and using the fact that the derivative of the bending moment is the shear force, we obtain

$$
V(x)=\frac{q(\alpha-2)(\alpha-1)}{\Gamma(\alpha)}\left(-x^{\alpha-3}+\frac{L^{\alpha-3}}{2^{\alpha-3}}\right) .
$$

Also for $\alpha=4$, the original integer order expression for the shear force is recovered.

\subsection{The shear effects of the Timoshenko-Ehrenfest beam theory}

From the TE beam theory [1, for cross sections suffering small distortions, without losing linearity, the shear deformation is given by the first order ordinary differential equation

$$
\frac{d v}{d x}=-c \frac{V}{G A},
$$

where $v=v(x), V=V(x), G$ is the cross elasticity modulus, and $c$ is the shear coefficient related to the bending deformation at the height of the section, which depends on its geometric dimensions and shape. 
In Eq. 12 , $A$ is the cross-sectional area, $G=\frac{E}{2(1+\nu)}$ for rectangular sections (our case study), and $c=\frac{12+11 \nu}{10(1+\nu)}$, where $\nu$ is a Poisson module.

Solving Eq.(12) considering $V$ given in Eq.(11) for $\alpha=4$, we obtain

$$
v_{c}(x)=\frac{q c}{G A}\left(\frac{x^{2}}{2}-\frac{L x}{2}\right) .
$$

Therefore, considering the bending and shear deformations, the final deflection can be given by means of the superposition principle, as below.

$$
v(x)=v_{f}(x)+v_{c}(x),
$$

where $v_{f}(x)$ and $v_{c}(x)$ are the bending and shear deformations, respectively in Eqs.(2) and (13).

Thus,

$$
v(x)=\frac{q}{E I}\left(\frac{-x^{4}}{24}+\frac{L x^{3}}{12}-\frac{L^{3} x}{24}\right)+\frac{q c}{G A}\left(\frac{x^{2}}{2}-\frac{L x}{2}\right) .
$$

The characterization of the effects seen in this section are addressed by TE beam theory, discussed in the following.

The TE model considers, in addition to bending deformation, the shear effects that the beam can undergo when subjected to loading. In this case, the cross sections of a beam carry out, besides the translational displacement (deflection) and the rotation purely associated with the bending accomplished by the $\mathrm{BE}$ model, the rotation around their barycenter with respect to the neutral plane, in this model no longer kept orthogonal. This additional rotation comes from the shear stresses more present in the extremities of thicker beams 1 and [3]. In essence, the first premise of EB's theory is preserved, but not the second. The resulting system of equations is

$$
\left\{\begin{array}{l}
\frac{d^{3} \theta}{d x^{3}}=\frac{q}{E I} \\
\frac{d v}{d x}=\theta-\frac{E I c}{G A} \frac{d^{2} \theta}{d x^{2}}
\end{array}\right.
$$

where the dependent variables of the $\theta=\theta(x)$ and $v=v(x)$ system represent, respectively, the cross section rotation and the deflection of the barycentric axis, therefore including the shear effects.

For the particular case of a simply supported under uniformly distributed load, the deflection results

$$
v(x)=\frac{q}{E I}\left(-\frac{x^{4}}{24}+\frac{L x^{3}}{12}-\frac{L^{3} x}{24}\right)+\frac{c q}{G A}\left(\frac{x^{2}}{2}-\frac{L x}{2}\right)
$$

for the boundary conditions $\theta\left(\frac{L}{2}\right)=0, M(0)=M(L)=0$ and $v(0)=0$.

Since Eq. 177) matches Eq.15, we propose in this work a fractional solution for the TE model.

\subsection{Fractional Timoshenko-Ehrenfest solution}

Using the superposition principle as stated in Eq.15, we propose a fractional solution for the TE equation as follows:

$$
v(x)=v_{F E B}(x)+v_{F C}(x),
$$

where $v_{F E B}(x)$ and $v_{F C}(x)$ are the fractional solutions for deflection (effects of bending moments) and shear, respectively. 
The solution $v_{F E B}(x)$ was obtained in Eq. (8). On the other hand, we obtain $v_{F C}(x)$, integrating Eq. (12), using the fact that the shear force integral $V$ is the bending moment $M$, and finally we consider $M$ as the fractional bending moment given in Eq. (9). From that, we get

$$
v_{F C}(x)=\frac{c}{G A} \frac{q(\alpha-1)}{\Gamma(\alpha)}\left(x^{\alpha-2}-L^{\alpha-3} x\right) .
$$

Finally, taking Eq.(19) and Eq.(8) in Eq.(18), we have

$$
\begin{aligned}
v(x) & =\frac{q}{E I}\left[\frac{-x^{\alpha}}{\Gamma(\alpha+1)}+\frac{4 L^{\alpha-3}}{2^{\alpha-1} \Gamma(\alpha+1)}\left(2^{\alpha-1}-\alpha\right) x^{3}+\frac{L^{\alpha-1}}{2^{\alpha-1} \Gamma(\alpha+1)}\left(4 \alpha-3.2^{\alpha-1}\right) x\right] \\
& +\frac{c}{G A} \frac{q(\alpha-1)}{\Gamma(\alpha)}\left[x^{\alpha-2}-L^{\alpha-3} x\right],
\end{aligned}
$$

which is the fractional TE solution that provides the deflection suffered by a simply supported beam under an uniformly distributed load.

For $\alpha=4$, Eq. 20 matches Eq. (17) and the solution is verified.

\section{Application and results}

Confronting Eqs. (8) and (20) against the Ansys results, the $\alpha$ values for the particular solutions of FEB and FTE, respectively, can be found. For the sake of clarity, we have conceived a simple example of structure loaded within the linear elastic limits: a simply supported AISI 1020 steel beam, isotropic, prismatic, with dimensions $(b, h, L)=(0,3 m ; 0,8 m ; 5 m)$, and subjected to an uniformly distributed transversal load $q=1 \times 10^{4} \mathrm{~N} / \mathrm{m}$. According to Ansys simulation, the maximum stress for such a load is $\sigma_{\max }=3,1996 \times 10^{6} \mathrm{~Pa}$, hence far below the maximum yield stress for this material, on average $\sigma_{\max }=4,2 \times 10^{8} \mathrm{~Pa}$ 7.

As the variable in question is the transversal deflection, from symmetry, the maximum deflection locates in the middle of the beam, i.e. $x=2,5 \mathrm{~m}$. We have chosen this position to obtain the fractional order $\alpha$ because of its relevance to prevent failure. From Ansys, the maximum deflection is $3,5609 \times 10^{-5} \mathrm{~m}$. Then, the resulting outcome is $\alpha=4,1585$ for FEB and $\alpha=4,0876$ for FTE, as expected, as the closer $\alpha$ is to 4, the less influential is the fractional order "needed" for adjustment toward the problem under investigation (accurately modeled in Ansys). This means that the original integer order TE model is confirmed closer to the actual real structure behavior, since the interference from the fractional adjustment in FTE is lower than for the case of the original integer order EB model; this is coherent with the fact that the TE model encompasses the shear effects, on the contrary of EB, and represents better the real structure behavior. Employing these $\alpha$ values back into Eqs. (8) and (20), correspondingly, the particular fractional solutions are achieved. From this point onward the final equations for deflections can be compared with the Ansys response along the entire beam.

Figure 1 reveals the deflection behavior of EB, FEB, TE and FTE solutions with respect to Ansys. Besides, we have included the deflection ratio between FBE and TE solutions (in this case, $\alpha=4,0614)$. All five graphs are symmetrical and show progressively larger differences from the middle to each extremity, which is in accordance with the shear effects, that lead the structure to an increased transversal deflection to accommodate those shear deformations. For this reason in the Ansys model, the closest to reality, the deflection is the highest; almost as much as it would be in an experiment, all structural relaxation tend to be contemplated in the numerical model. Still with respect to Ansys, from the worst to the best are the graphs EB, TE, FEB and FTE, behaving as expected. EB lacks on the shear effects, and that is why it is significantly worse (far from Ansys) than TE; on the other hand, when comparing FEB and FTE, the original shear effects 
consideration in TE still makes FTE prevail over FEB, but very slightly. The latter shows that the fractional calculus approach is capable of achieving a good model adjustment not depending importantly on the accuracy of the original integer order model (at least for this application). Besides, from the comparison between FEB and TE, as the first is closer to Ansys, FEB surely incorporates the shear effects originally absent in EB, and as the results suggest, possibly other physical effects.

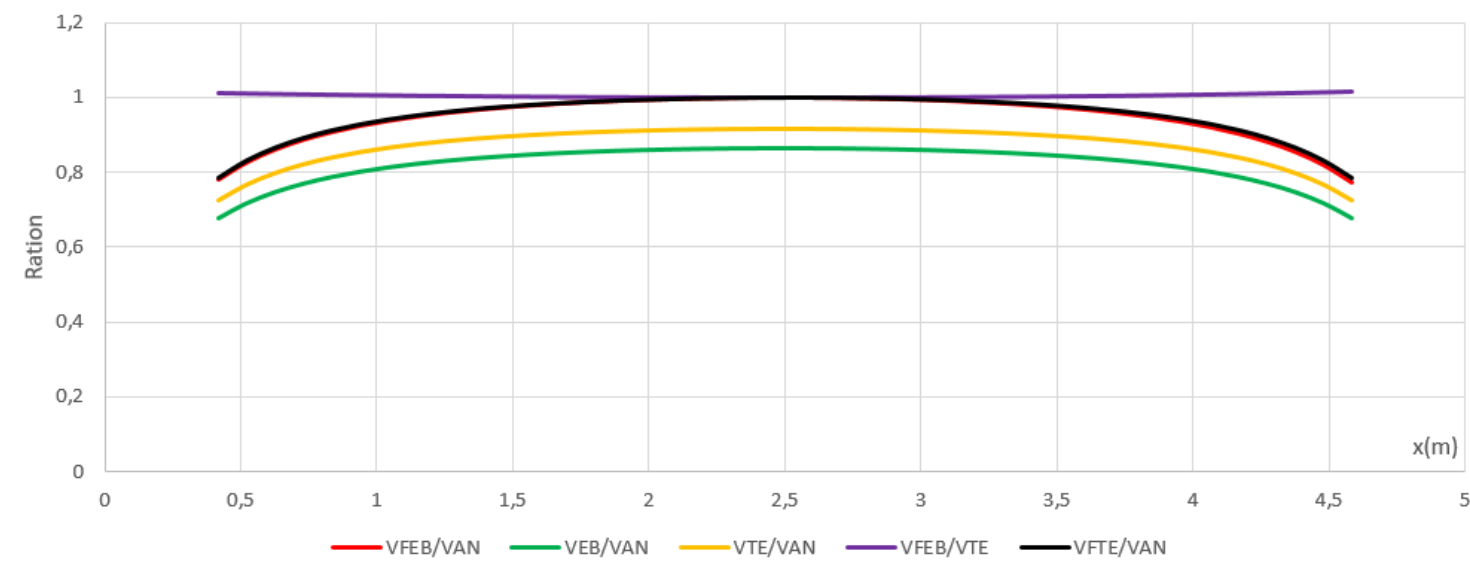

Figure 1: Deflection ratio for the solutions of the integer and fractional order with respect Ansys, and deflection ratio for the solutions of FEB with respect to TE.

\section{Conclusions}

In this work, we have proposed fractional models for the transversal deflection of Euler-Bernoulli and Timoshenko-Ehrenfest beams. We have obtained the respective fractional solutions considering the case of a simply supported beam subjected to an uniformly distributed static loading. From the analysis of a specific example, the fractional solutions have converged to a close approximation to the corresponding Ansys model of reference. The fractional Euler-Bernoulli solution performed better than the original integer order Timoshenko-Ehrenfest solution, which ensures the accomplishment of the shear effects -absent in the original integer order Euler-Bernoulli solution. Besides, this solution suggests the accomplishment of other physical effects as well, since it approaches closer to the predicted real structure behavior. Both fractional solutions are novelties as far as we know. From the analysis of those equations using the Ansys model as reference, this investigation may lead to identify the repercussion of different physical effects present within the structure in the magnitude of the fractional order. 


\section{References}

[1] Assan, A. E. Resistência dos Materiais-Volume 1, 1a. edição. UNICAMP, Campinas, 2010.

[2] Camargo, R. F. and Oliveira, E. C. Cálculo Fracionário, 1a. edição. Editora Livraria da Física, São Paulo, 2015.

[3] Fleishfresser, S. A. Uma formulação do método dos elementos de contorno para a análise de vigas de Timoshenko, Tese de Doutorado, UFPR, Curitiba, 2012.

[4] Herrmann, R. Fractional Calculus: An Introduction for Physicists, 2nd. edition. World Scientific, GigaHedron, 2014.

[5] Ribbeler, R. C. Resistência dos Materiais, 5a. edição. Pearson, São Paulo, 2004.

[6] Teodoro, G. S. Cálculo fracionário e as funções de Mittag-Leffler, Dissertação de Mestrado, Unicamp, Campinas, 2014.

[7] Timoshenko, S. P., James, E. G. Mecânica dos Sólidos, 1a. edição traduzida. LTC-Livros Técnicos e Científicos, Rio de Janeiro, 1983. 